\title{
TTR
}

Traduction, terminologie, re?daction

\section{Droit, traduction, langue et idéologie : Kata poda ou la traduction pas à pas selon Justinien $1^{\text {er }}$ \\ Law, translation, language, and ideology: Kata poda or translation step by step according to Justinian the $1^{\text {st }}$}

\section{Claire-Hélène Lavigne}

Volume 18, numéro 1, 1er semestre 2005

Traductions et représentations : Parcours dans l'espace hispanique II Translations and Representations: Exploring the Hispanic World II

URI : https://id.erudit.org/iderudit/014372ar

DOI : https://doi.org/10.7202/014372ar

Aller au sommaire du numéro

Éditeur(s)

Association canadienne de traductologie

ISSN

0835-8443 (imprimé)

1708-2188 (numérique)

Découvrir la revue

Citer cet article

Lavigne, C.-H. (2005). Droit, traduction, langue et idéologie : Kata poda ou la traduction pas à pas selon Justinien $1^{\mathrm{er}}$. TTR, 18(1), 183-202.

https://doi.org/10.7202/014372ar
Résumé de l'article

Cet article porte sur la Constitutio Tanta 21 qui interdit la rédaction de commentaires si ce n'est la traduction ultra-littérale du latin vers le grec qui frappe le Digeste de Justinien. Il débute par une analyse de la teneur et de la portée de Tanta 21, puis il s'intéresse aux divers facteurs qui ont pu participer à la formulation de cette interdiction, c'est-à-dire les principes appliqués à la traduction biblique, l'influence de la tradition juridique romaine et finalement le statut du latin comme langue juridique par excellence. 


\section{Droit, traduction, langue et idéologie : Kata poda ou la traduction pas à pas selon Justinien $\mathbf{1}^{\text {er }}$}

\section{Claire-Hélène Lavigne}

Statuam hastam auram tenentem

Quintillien

Il est tautologique d'affirmer, de nos jours, que les lois doivent être rédigées dans la langue des justiciables. Historiquement, ce ne fut toutefois pas toujours le cas. À titre d'exemple, l'Edictum Theoderici (après 458$)^{1}$ et le Liber judiciorum $(654)^{2}$ ont été rédigés dans une langue, le latin, qui n'était pas le vernaculaire du peuple. Dans le même ordre d'idées, le Corpus Iuris Civilis $(C I C)^{3}$ a été compilé en latin à une

\footnotetext{
${ }^{1}$ Au sujet de l’Édit, voir entre autres : Vismara (1992), pp. 275-315.

${ }^{2}$ Au sujet du Liber, voir entre autres : The Visigothic Code (Forum judicum) (1982).

${ }^{3}$ Le Corpus Iuris Civilis est un ensemble de textes juridiques rédigés en latin qui a été compilé au Haut Moyen Âge à l'instigation de l'empereur byzantin Justinien $1^{\mathrm{er}}$ (482-565). Le Corpus, comme son nom l'indique, est constitué de cinq textes distincts (le Codex constitutionum, le Digeste ou les Pandectes, les Institutes, le Codex Repetitae Praelectionis et les Novellae Constitutiones ou Novelles) qui formaient, avec les Cinquante Décisions, l'ensemble du droit de l'empire romain d'Orient. Le Corpus, à l'exception des Novelles, n'est pas un texte original. Il est composé principalement des écrits de divers jurisconsultes ayant vécu aux $\mathrm{I}^{\mathrm{e}}, \mathrm{II}^{\mathrm{e}}$ ou $\mathrm{III}^{\mathrm{e}}$ siècles. Les écrits ont été regroupés, corrigés et adaptés au $\mathrm{VI}^{\mathrm{e}}$ siècle par les commissions chargées de les compiler. Le Codex constitutionum fut compilé conformément aux instructions de Justinien $1^{\mathrm{er}}$. Il a
} 
été commandé à Tribonien (?-545) en 528. Ce dernier et dix collaborateurs devaient faire une compilation des diverses constitutions impériales présentes dans les codes existants (codes Grégorien [? 291-292 ?], Hermogénien [293294]) ainsi que des constitutions promulguées depuis Théodose II. La commission devait omettre les textes qui étaient désuets, corriger et amender les documents lorsque nécessaire et clarifier les termes et les expressions employés lorsqu'ils étaient obscurs. Les constitutions datant de 438 au règne de Justinien devaient également être incluses dans le Codex constitutionum. Les constitutions dûment corrigées ont été classifiées selon leurs thèmes et réunies dans le Codex constitutionum. Celui-ci fut publié le 7 avril 529. En 533, le Codex constituriorem était devenu désuet, cela à cause de la grande activité législative des commissions instituées par Justinien depuis 529. Ce dernier chargea Tribonien et cinq autres jurisconsultes de constituer une nouvelle édition revue et corrigée du code, ce qui devait le rendre conforme au Digeste et aux Institutes. Le Codex Repetitae Praelectionis fut promulgué en 534. Il est divisé en douze livres qui sont eux-mêmes subdivisés en titres. L'ordre de présentation des divers domaines de droit est le suivant : le droit canonique, les sources du droit, les responsabilités des hauts fonctionnaires, le droit privé, le droit pénal et le droit administratif. Le Digeste, également nommé Pandectes, est le plus grand des quatre volumes qui forment le Corpus Iuris Civilis. Il a été commandé en 530 à Tribonien et à une commission composée de 16 jurisconsultes. Ce recueil est une compilation des écrits révisés de 39 prudentes. Les compilateurs du Digeste devaient éliminer les contradictions entre les divers écrits, les corriger et les mettre à jour. Les opinions juridiques ainsi corrigées ont été réunies en 50 livres qui sont eux-mêmes subdivisés en titres - ce qui explique le nom du volume, car le terme digeste signifie distribution sous titre. Le Digeste fut promulgué le 16 décembre 533 par deux constitutions, l'une écrite en latin, l'autre en grec. Les Novellae Constitutiones post codicem sont des ordonnances particulières faites par Justinien afin de clarifier, d'expliquer ou d'abroger certains passages ou articles du Codex Repetitae Praelectionis ou du Digeste. On compte 168 ordonnances dont 160 auraient été rédigées par Justinien. Les Novelles ont presque toutes été écrites en grec. Il existe une traduction en latin de ce texte juridique qui aurait été probablement faite au $\mathrm{VI}^{\mathrm{e}}$ siècle, soit après la mort de Justinien. Les Institutes sont un texte législatif que Justinien destinait à l'enseignement. Elles ont été composées concurremment au Digeste par Tribonien, Dorothée (Dorotheus) et Théophile (Théophilus). Bien que ce texte ait été publié en novembre 533, il n'eut force de loi qu'en décembre de la même année, c'est-à-dire en même temps que le Digeste. Les Institutes sont composées, en majeure partie, d'extraits de jurisconsultes classiques. Contrairement au Digeste, il n'y a pas, dans le corps du texte, de renvois ou d'indication de la provenance ou de l'auteur des extraits. Les compilateurs semblent avoir pris certains passages directement du Digeste. Il y a également dans le texte des renvois ou des extraits d'un certain nombre de constitutions impériales. D'autres passages proviendraient des Institutes de Gaius ou de ses res cottidianae, des Institutes 
époque et dans une société, l'empire romain d'Orient, où cette langue n'était plus comprise par la majorité des gens, y compris bon nombre d'étudiants en droit et de juristes ${ }^{4}$. Dans le cas de l'Edictum Theoderici et du Liber judiciorum l'explication se trouve fort probablement dans le rapport entre langue du savoir (latin) et langue vernaculaire, alors que dans celui du CIC l'explication n'est pas si facile. En effet, non seulement le $C I C$ est rédigé en latin alors que la langue de la majorité est le grec, mais encore le Digeste, l'un des quatre textes qui le composent, est frappé d'une interdiction de rédiger des commentaires : seules la traduction littérale du latin vers le grec et la rédaction de paratitla sont permises ${ }^{5}$. Pourtant le grec est, tout comme le latin et l'hébreu, une langue prestigieuse. En effet, ces trois langues sont considérées comme les langues du savoir séculier et religieux. Les nombreuses versions grecques de l'Ancien Testament réalisées avant le III $^{\mathrm{e}}$ siècle de notre ère que ce soit la Septante ${ }^{6}$, les versions d'Aquila ${ }^{7}$ (v. 130), de Théodotion ${ }^{8}$ (avant la fin du $\mathrm{II}^{\mathrm{e}}$ siècle), de Symmaque (vers 170 ) et des versions Quinta et Sexta telles que nommées par Origène

de Florentinus, des Institutes de Marcien, des Institutes d'Ulpien, des Institutes de Paul, des libri VII regularum d'Ulpien et finalement des libri differentiarum de Modestin. En ce qui concerne la présentation, les Institutes de Gaius ont servi de base dans l'établissement du plan des Institutes de Justinien. Ces dernières sont composées d'une constitution préliminaire (Prooemium) et de quatre livres eux-mêmes subdivisés en titres. Chaque titre comprend un paragraphe d'introduction et un certain nombre d'articles.

${ }^{4}$ Le Digeste, tout comme les Institutes d'ailleurs, devait servir, entre autres, à l'enseignement du droit. Voir Stein (1999), p. 35. Concernant l'enseignement du droit dans l'Antiquité, voir entre autres : Chorus (1993), pp. 195-204.

${ }^{5}$ L'empire byzantin était, au temps de Justinien, multilingue. La langue de la majorité était le grec, mais on y parlait également le syriaque, le coptic, l'arménien, le géorgien, l'arabe, le persan, l’hébreu, etc. Seul le Digeste est frappé d'une interdiction de rédaction de commentaires ou de traduction littérale du latin vers le grec. Il existe une paraphrase (Paraphrase de Théophile) des Institutes, quelques commentaires sur les Novelles et plusieurs sur le Codex. Voir entre autres : Gaudemet (2000).

${ }^{6}$ Au sujet de la Septante, voir Harl et al. (1988).

${ }^{7}$ Au sujet de la version d’Aquila, voir entre autres : Barthélemy (1963).

${ }^{8}$ Au sujet de la version de Théodotion, voir O’Connell (1972). 
dans son Hexaplorum ${ }^{9}$ confirment cet état de fait ${ }^{10}$. D’ailleurs, Justinien connaissait la Septante et la version d'Aquila. Il y fait spécifiquement référence dans la Novelle 146. Cette dernière autorise l'utilisation, dans les synagogues, de ces traductions grecques ou de toutes autres versions réalisées dans une autre langue. Ainsi, pourquoi interdire, dans le cas du Digeste, la traduction autre que littérale du latin vers le grec ou la rédaction de commentaires ? Cet article débute donc par une brève analyse de la teneur et de la portée de l'article 21 (constitutio Tanta $(\text { Tanta 21) })^{11}$, puis il s'intéressera à certains facteurs qui ont pu participer à la formulation de l'interdiction de traduction autre que littérale du latin vers le grec ${ }^{12}$ qui frappe le Digeste.

\section{Interdiction contenue dans Tanta 21 1.1 Libellé de Tanta $21^{13}$}

Tanta 21 est formulé en ces termes :

"Hoc autem quod et ab initio nobis visum est, cum hoc opus fieri deo adnuente mandabamus, tempestivum nobis videtur et in praesenti sancire, ut nemo neque eorum, qui in praesenti iuris peritiam habent, nec qui postea fuerint audeat commentarios isdem legibus adnectere: nisi tantum si velit eas in Graecam vocem transformare sub eodem ordine eaque consequentia, sub qua voces Romanae positae sunt (hoc quod Graeci kata poda

${ }^{9}$ Origen (1875).

${ }^{10}$ Nous reviendrons sur le lien entre la langue grecque et la traduction de textes religieux ou juridiques après avoir analysé la portée de Tanta 21.

${ }^{11}$ Il existe de nombreux articles et ouvrages portant sur l'interdiction de rédiger des commentaires ou sur son non-respect par les juristes. Voir entre autres : De Robertis (1984), Van der Wal (1953), Wenger (1953), Berger (1945), Pringsheim (1950), pp. 383-415. Au sujet, du non-respect de Tanta 21 à la Renaissance, voir, entre autres, Maclean (1992), pp. 50-59. Toutefois, ils s’intéressent peu à l'interdiction de traduction autre que littérale.

12 L'interdiction est reprise dans quatre constitutions: De confirmatione Digestorum, (Tanta 21); Dedoken, (article 21); les deux autres se trouvent dans le livre 1, Titre XVII du Codex. Dans le cadre de cet article, nous utilisons le texte que l'on trouve dans le Digeste (De confirmatione Digestorum, Tanta 21).

${ }^{13}$ Les citations latines sont tirées de l'édition de Mommsen; la version française est tirée de l'ouvrage de Gaudemet (2000a) aux pages 314 à 321, note 16 . 
dicunt), et si qui forsitan per titulorum subtilitatem adnotare maluerint et ea quae paratitla nuncupantur componere. Alias autem legum interpretationes, immo magis perversiones eos iactare non concedimus, ne verbositas eorum aliquid legibus nostris adferat ex confusione dedecus. Quod et in antiquis edicti perpetui commentatoribus factum est, qui opus moderate confectum huc atque illuc in diversas sententias producentes in infinitum detraxerunt, ut paene omnem Romanam sanctionem esse confusam. Quos si passi non sumus, quemadmodum posteritatis admittatur vana discordia? Si quid autem tale facere ausi fuerint, ipsi quidem falsitatis rei constituantur, volumina autem eorum omnimodo corrumpentur. Si quid vero, ut supra dictum est, ambiguum fuerit visum, hoc ad imperiale culmen per iudices referatur et ex auctoritate Augusta manifestetur, cui soli concessum est leges et condere et interpretari. »

[D’autre part, ce qui nous était apparu dès le début, lorsque avec l'aide de Dieu nous avons ordonné d'entreprendre ce travail, il nous semble opportun de le prescrire à nouveau maintenant : à savoir qu'aucun des jurisconsultes de notre temps, ni ceux qui viendront par la suite n'osent adjoindre des commentaires à ces lois; nous permettrons seulement de les traduire en grec, selon le même plan et en respectant l'ordre dans lequel se suivent les mots latins (ce que les Grecs appellent suivre pied à pied) et de faire quelques sommaires sur chacun des titres, ce qu'on appelle des paratitles. Mais nous ne voulons pas qu'on fasse d'autres interprétations, qui sont plutôt des perversions du texte. Nous craignons que le verbiage des interprètes ne jette la confusion dans nos lois, ce qui est arrivé dans les anciens commentaires de l'édit perpétuel. Cet ouvrage sagement fait, ils l'ont déformé en en tirant des solutions contradictoires, si bien que presque toute la jurisprudence romaine s'est trouvée dans la plus grande confusion. Si nous n'avons pu souffrir ce fatras des anciens commentateurs, comment la postérité accepterait-elle ces vaines divergences ? Ceux qui auront la témérité de composer de pareils commentaires se rendront coupables du crime de faux, et leurs ouvrages seront détruits. Mais si, comme on l'a dit plus haut, quelque chose semble douteux, les juges en référeront à l'empereur, et le sens des lois sera fixé par l'autorité impériale à qui seule a été donné le droit de faire des lois et de les interpréter.] 


\subsection{Analyse de Tanta 21}

Tanta 21 suit l'ordre de présentation suivant : renvoi implicite à la constitution De Conceptione Digestorium (Deo auct. 12), formulation de l'interdiction et spécification des exceptions - qui sont au nombre de deux - fondement de l'interdiction, explication de la peine encourue pour quiconque transgresse l'interdiction ${ }^{14}$ et, finalement, établissement de l'autorité compétente pour interpréter le droit, c'est-à-dire l'empereur lui-même.

\subsection{Renvoi à Deo auct. 12}

Justinien débute en affirmant qu'il reprend l'interdiction qui avait été formulée en 530 lorsqu'il lança le processus de compilation du Digeste, ce qui n'est pas tout à fait exact. En effet, Deo auct. 12 interdit la rédaction de commentaires et permet les indices et les titulorum. Il n'y est fait, toutefois, aucune mention de la traduction. Il faut attendre l'adoption du Digeste, le 16 décembre 533, pour que le législateur la mentionne. Diverses hypothèses ont été formulées afin d'expliquer les raisons de ce changement d'attitude de la part de Justinien. Elles tendent toutefois vers l'impressionnisme. Pringsheim (1950, p. 392) explique que le législateur ne pouvait pas annoncer dans Deo auct. 12 un projet de législation en latin et en même temps en mentionner la traduction vers le grec. Il ne justifie ou n'explique d'aucune façon cette affirmation. De son côté, Berger (1945, p. 676) soumet que Justinien ne pouvait plus, lors de la promulgation du Digeste, ignorer le «fait grec », ce qui l'obligeait à prévoir la traduction de ces textes législatifs. Il n'explique toutefois pas pourquoi Justinien pouvait taire la traduction lors de la formulation du projet et qu'il devait la prévoir lors de la promulgation du texte. De plus, même si cette affirmation de Berger est fondée, pourquoi Justinien n'a-t-il pas tout simplement ordonné une traduction officielle de ces textes, quitte à ne donner force de loi qu'à l'une des deux versions ${ }^{15}$ ? Ces hypothèses sont insuffisantes, selon nous, pour expliquer l'interdiction de traduction autre que littérale qui

${ }^{14}$ Quiconque transgresse Tanta 21 sera trouvé coupable de contrefaçon, sera déporté, l'ensemble de ses biens sera saisi et tout ouvrage qu'il aura produit en contravention de cette règle sera détruit (D 48.10.1.13, poena falsi).

${ }^{15}$ Watson (1988, p. 140) affirme qu’à aucun moment Justinien n’a eu l'intention de faire traduire en grec les textes du CIC. 
frappe le Digeste. Nous reviendrons sur ce point dans la seconde partie de cet article.

\subsection{Formulation de l'interdiction et spécification des exceptions}

Retournons, pour l'instant, à l'analyse de Tanta 21. Le deuxième élément abordé dans cet article consiste en l'interdiction de rédiger des commentaires sur le texte du Digeste si ce n'est de le traduire pas à pas vers la langue grecque.

Justinien énonce la règle interdisant à tout juriste - présent ou futur - d'annexer des commentaires au Digeste, puis spécifie les exceptions, c'est-à-dire, premièrement, que la traduction du latin vers le grec est permise en autant qu'elle suive le latin "pas à pas " (kata poda) et, deuxièmement, l'insertion de notes explicatives (paratitla), puis Justinien réitère l'interdiction concernant toute autre interprétation. Deux aspects méritent d'être notés. Le premier a trait à l'utilisation de l'expression grecque kata poda, le second porte sur la formulation de la restriction.

En ce qui concerne l'expression grecque kata poda, nous avons tenté d'établir une chaîne de citations de son utilisation dans un cadre traductionnel ${ }^{16}$. Nous n'avons trouvé aucune occurrence, dans l'empire romain d'Orient ou dans la Grèce Antique, de l'expression kata poda prise dans le sens de "traduction pas à pas » ou de «traduction mot à mot». Kata poda ne semble donc pas être une expression consacrée pour désigner une méthode littérale de traduction. Certes, Xénophon, Hérodote et Thucydide, entre autres, utilisent cette expression, mais dans le sens de « suivre au pied » ou de "suivre à la trace $»^{17}$. Justinien a peut-être créé un néologisme sémantique lorsqu’il

\footnotetext{
${ }^{16}$ L'auteure tient à remercier Effrosyni Fragkou pour ses recherches sur les chaînes de citations.

${ }^{17}$ Voici une occurrence de l'utilisation par Xénophon de l'expression kata podas dans Hellinica: "hoi d'Athênaioi kata podas pleontes hôrmisanto tês Cherronêsou en Elaiounti nausin ogdoêkonta kai hekaton. entautha dê aristopoioumenois autois angelletai ta peri Lampsakon, kai euthus anêchthêsan eis Sêston ». Source : http://www.perseus.tufts.edu Kata podas est utilisé dans le contexte de bataille navale et signifie « suivre au pied ».
} 
a appliqué kata poda à la traduction littérale d'un texte juridique, mais il est difficile de l'affirmer.

Le second aspect, avons-nous dit, porte sur la formulation de Tanta 21. Cet article semble identifier la traduction, qu'elle soit littérale ou libre, à un procédé de rédaction de commentaires, donc, à l'exégèse. La formulation retenue dans Tanta 21 confirme en quelque sorte cette interprétation. En effet, le texte latin commence par interdire tout commentaire, puis il retire du champ d'application de Tanta 21, par l'utilisation de l'expression latine nisi tantum (si ce n'est), la traduction littérale vers le grec. Ainsi, dans l'optique justinienne, il fallait exclure spécifiquement la traduction littérale du champ d'application de Tanta 21 sans quoi toute traduction du Digeste aurait été comprise comme interdite.

\subsection{Fondement de l'interdiction et autorité compétente}

Le troisième élément touché par Tanta 21 est consacré au fondement de l'interdiction. Trois points méritent ici d'être analysés. Le premier porte sur l'utilisation par Justinien du terme perversiones; le second sur la raison de l'interdiction, alors que le troisième fournit une justification à l'interdiction. En ce qui concerne le premier point, le législateur, lorsqu'il utilise le terme perversiones pour justifier son interdiction d'interprétation du texte associe ce mot au terme interpretationes. Perversiones a ici le sens de «renversement de la construction d'une phrase ou des mots ». On peut ainsi voir que Justinien interdit non seulement le renversement ou la transformation du sens (interpretationes), mais encore qu'il prohibe tout changement à l'ordre des mots ou des phrases (perversiones) restant ainsi cohérent avec son utilisation de l'expression grecque kata poda.

Le second aspect explique que l'interdiction veut empêcher que la compilation ne devienne chaotique à cause de la multiplication des interpretationes ou des perversiones. On voit ici que le but avoué de l'interdiction et de la restriction de traduction est d'empêcher le foisonnement des réseaux de signification du texte. Justinien semble être conscient que le texte est toujours ouvert à interprétation et que son réseau de signification est en perpétuelle mouvance. Le troisième et

En ce qui concerne l'utilisation d'expressions grecques, de noms propres grecs et des notions de philosophie grecque dans le CIC, voir entre autres : Winkel (1997), plus particulièrement p. 377. Voir également Troje (1971), p. 12 sq. 
dernier point justifie, par le jeu d'une anecdote historique, l'interdiction de rédaction de commentaires et de traduction autre que littérale du latin vers le grec. Le législateur affirme que l'Édit perpétuel fut l'objet de si nombreuses et diverses interprétations que le système juridique romain en devint presque inopérant. Justinien semble ainsi vouloir préserver l'intégrité de la lettre et du sens de sa compilation.

Le dernier aspect traité par Tanta 21 énonce que seul Justinien a autorité pour interpréter, en cas d'ambiguïté (ambiguitas), le Digeste. Les stratégies d'interprétation des écrits sont développées dans l'Antiquité romaine dans le cadre des débats judiciaires. L’herméneutique telle qu'elle est utilisée par les anciens (Cicéron, Quintillien, etc.) s'inscrit dans un mouvement antagoniste. Cicéron lorsqu'il inclut la théorie herméneutique dans la théorie rhétoricienne, comprend l'interprétation en termes de controverse. Selon cet auteur, la controverse dans un écrit peut prendre trois formes: l'intention de l'auteur ne concorde pas avec le texte écrit (scriptum c. voluntas ou scriptum c. sententia); un terme ou un passage de l'écrit est ambigu (ambiguitas); deux sections de l'écrit ou deux écrits complémentaires se contredisent (ex contrariis legibus). De plus, Cicéron définit la rhétorique, dans son sens large, comme étant l'art de persuader; cet art se concrétise dans la pratique - que ce soit pour l'étudiant ou le juriste - de la procédure nommée disputatio in utramque partem (argumentation pour et contre un point). L'interprétation en tant que partie de la rhétorique partage cette technique avec la rhétorique. L'idée de controverse présente dans l'herméneutique cicéronienne repose sur la possibilité qu'a l'interprète de favoriser soit le scriptum (lettre), soit la voluntas/sententia (volonté/esprit), cela dans le but de démontrer la justesse de son argumentation. Ainsi, celui qui prône le respect du scriptum afin de préserver l'intention de l'auteur de l'écrit doit insister sur le fait qu'en respectant la lettre, il respecte l'intention de l'auteur, alors que celui qui appuie son raisonnement sur la voluntas doit soit interpréter le scriptum de telle manière qu'il confirme la voluntas, ou encore établir que le texte est ambigu, transférant par le fait même la controverse entre le scriptum et la voluntas à l'ambiguïté (ambiguitas). Notons que ces deux catégories de controverse sont distinctes l'une de l'autre. En rhétorique, l'opposition scriptum/voluntas se rattache au moyen de preuve (fondement juridique), alors que l'ambiguïté (ambiguitas), la deuxième cause de controverse, est une question de style. Ainsi, la rhétorique caractérise la signification différemment selon ses divisions: sous l'inventio, la signification repose dans l'intention de l'auteur, alors que sous l'elocutio, la signification repose 
dans le sens des mots. L'interprète a toujours la possibilité de contrer l'interprétation stricte de l'écrit (scriptum) en ayant recours à l'équité, qui devient un outil fondamental de l'interprétation. Il est ainsi possible d'interpréter le texte de façon équitable afin de s’opposer à la lettre. En faisant appel à l'équité, on interprète contra scriptum, c'est-à-dire contre une interprétation stricte, mais on peut également interpréter supra scriptum en faisant appel au syllogisme, en ce sens que l'on prend en considération ce qui n'est pas dans le texte. On reconnaît ainsi l'impossibilité fondamentale pour un écrit de s'appliquer à toutes les circonstances et de conserver une signification à travers le temps. On admet que le texte, afin de survivre au passage du temps, doit être mouvant et ouvert à l'interprétation. Cette stratégie de corriger ou d'adapter le texte aux circonstances particulières de sa réception représente un des outils les plus importants de la tradition de l'interpretatio scripti, et, de façon subsidiaire, de la rhétorique ${ }^{18}$.

Ainsi, Justinien, afin de contrer le modèle rhétoricien d'interprétation du texte juridique, se réserve l'exclusivité de dire le droit, ce qui lui permet de restreindre le nombre d'interprètes du texte et d'en garantir l'autorité. En effet, tout mouvement interprétatif pose le problème de l'autorité et de la préservation du texte contre l'hérésie. L'interdiction de rédaction de commentaires et la restriction de traduction donnent un pouvoir à l'interprétation justinienne, une force qui tire son autorité de l'exclusion de toute autre interprétation. Elle se veut à ce moment définitive, immuable et unique; elle seule détient la " vérité ». Justinien a donc une vision « extrêmement réductrice du rôle de la doctrine et du processus interprétatif » (Morin, 2004, p. 80).

Justinien semble associer la traduction sous toutes ses formes à la rédaction de commentaires et ce, à cause du libellé de Tanta 21. Toutefois, la traduction kata poda est permise puisqu'on semble présumer qu'elle n'ajoute rien ${ }^{19}$; ce qui est donc proscrit, c'est l'ajout : tout ce qui n'est pas reproduction ultra-littérale de la lettre latine. Contrairement à la rédaction de commentaires et à la traduction qui

${ }^{18}$ Voir, entre autres, à ce sujet : Eden (1997).

${ }^{19}$ En parlant de la traduction littérale dans l’optique justinienne, Sarcevic (1997, p. 25) la nomme « strict litteral translation » et l'oppose à la « litteral translation ». Cette dernière, contrairement à la «strict litteral translation », permettrait d'apporter des changements syntaxiques afin de respecter les règles de grammaire de la langue d'arrivée et d'en faciliter la compréhension, sans pour autant s’éloigner du texte de départ. 
n'est pas ultra-littérale, la traduction kata poda ne serait pas un acte de production de sens nouveau dans l'optique justinienne, ce qui semble d'ailleurs être confirmé par la non-restriction du nombre de traductions qui peuvent être faites; seule la méthode de traduction qui doit être appliquée au texte fait l'objet d'une restriction. On voit ici poindre une présomption voulant que toutes traductions kata poda soient nécessairement identiques. Ainsi, dans la logique justinienne, la multiplication possible du nombre de versions ultra-littérales ne semble pas représenter un risque de glissement de sens ou de multiplication du réseau de signification. A contrario, Justinien reconnaît donc que la traduction, quand elle n'est pas ultra-littérale, se rapproche du mouvement interprétatif, qu'elle risque de provoquer la différence et est nécessairement un acte qui met de l'avant la force d'invention du traducteur/commentateur qui peut découvrir, ouvrir et s'approprier le texte. À ce moment, la traduction peut être assimilée à un acte rhétoricien. Voilà pourquoi Justinien n'autorise que la traduction kata poda. On découvre ici une conception concernant le langage et la façon dont il signifie. En effet, Justinien lorsqu'il interdit toute autre forme de traduction que la traduction ultra-littérale admet en quelque sorte que tout texte est composé d'un réseau de signification qui risque, lors du mouvement herméneutique, de donner lieu à diverses interprétations, ce qu'il veut à tout prix éviter.

\section{Traduction biblique et traduction juridique : idéologie 2.1 Idéologie}

Sarcevic (1997, p. 24 sq.), reprise entre autres par Zhao Yuhong (2000, p. 21-22), soumet que l'interdiction de traduction autre que littérale du latin vers le grec prendrait sa source dans la traduction biblique. L'interdiction de Justinien semble, en effet, indiquer un "malaise sémiotique " semblable à celui qui hantait, entre autres, Origène, Augustin $^{20}$ et Jérôme au moment de l'exégèse ou de la traduction biblique. Selon Augustin, le rôle de la traduction littérale est de récupérer une certitude originale qui n’a pas été obscurcie par les conventions humaines de la rhétorique; alors que pour Jérôme, la signification du texte est inviolable. Cette présomption définit également le programme de la traduction non sacrée de Jérôme. Contrairement à Cicéron et à Horace, Jérôme comprend la signification et le discours comme étant deux fonctions séparées. Il inverse, tout en

\footnotetext{
${ }^{20}$ Au sujet des «théories du langage » d'Origène et d'Augustin, voir entre autres, Martin Irvine (1994).
} 
utilisant leurs commentaires, les priorités de Cicéron et d'Horace. Ces derniers comprenaient le langage comme un outil facilitant la différence, alors que Jérôme voit le langage comme empêchant la fidélité.

La formule cicéronienne non verbum pro verbo est comprise par Jérôme comme un modèle de fidélité textuelle et non plus comme un modèle facilitant l'altérité. Le commentaire cicéronien est donc inversé afin de privilégier la signification et sera repris tout au long du Moyen Âge. S'inspirant de Jérôme, Boèce s'approprie le commentaire d'Horace. Ce faisant, il réhabilite l'utilisation de la traduction littérale pour les textes séculiers. En rejetant la traduction du sens, il redonne indirectement un motif rhétoricien à ce style de traduction en introduisant le mode rhétoricien de signification, en transposant et en introduisant la capacité linguistique d'un grand nombre de significations par le jeu de l'ambiguïté. Les rhetores latini minores et la tradition patristique, tout en utilisant les commentaires romains sur la traduction, rejettent le fondement rhétoricien sur lequel ils reposent. En effet, ils voient la traduction non pas comme devant faciliter et encourager la différence, mais plutôt comme permettant de récupérer une signification unique et immuable présente dans l'écrit et qui n’est pas dépendante du langage. ${ }^{21}$

Tout mouvement exégétique et traductionnel ${ }^{22}$, qu'il porte sur le texte biblique ou non, produit nécessairement un texte secondaire qui se présente comme véhiculant la signification et le sens véritable du texte primaire. Toutefois, l'exégèse n'est jamais complète en soi. Il reste toujours du sens à explorer, à faire dire au texte. La polysémie du texte n'est jamais «usée », ce qui demande obligatoirement de nouvelles interprétations, donc de nouveaux textes (Irvine, 1994, p. 252 sq.). Dans l'optique justinienne, ce qui pose problème dans cette multiplication interprétative des textes, c'est la question de la préservation de l'autorité, de l'unicité du texte, de sa perfection présumée et de l'orthodoxie de l'interprétation. En ce qui concerne le texte biblique, on garantit sa sauvegarde en présumant que l'écrit biblique détient une signification divine pré-linguistique qui est immuable et qui ne peut être corrompue par l'exégèse ou la traduction,

${ }^{21}$ À ce sujet, voir, entre autres, Copeland, (1989), pp. 15-35 ; (1991), p. 51 sq.

${ }^{22}$ Rappelons que selon la formulation de Tanta 21, Justinien associe la traduction à la rédaction de commentaires et donc à l'exégèse. 
alors qu'en ce qui concerne le texte de Justinien, son unicité et son autorité est préservée par Tanta 21. Il est ainsi possible que Justinien ait voulu transférer au texte juridique les principes de traduction et d'exégèse appliqués à la Bible. Toutefois, ce n'est, selon nous, qu'un des facteurs qui a participé à la formulation de Tanta 21. Il en existe au moins deux autres qui sont tout aussi importants. Le premier concerne le droit byzantin et ses conditions de formation, le second porte sur le statut du latin face au grec.

\subsection{Droit et langue}

Le droit byzantin ${ }^{23}$ prend sa source dans le droit romain, droit écrit qui s'exprime en latin. Le droit de l'empire romain d'Orient conservera, tout au long de son histoire, certaines caractéristiques propres au droit romain, entre autres : la dominance de son droit écrit ${ }^{24}$ et son pluralisme juridique. La première caractéristique "s'inscrit dans la tradition romaine impériale et domine pendant toutes les périodes de l'histoire du droit byzantin " (I. Tsourka-Papastathi, 1994, p. 610). Selon la tradition romaine ${ }^{25}$, l'empereur est la source vivante du droit écrit et séculier. Il est le seul à pouvoir interpréter, modifier ou abroger le droit séculier. Ce principe atteindra d'ailleurs son apogée dans le droit de Justinien, c'est-à-dire dans Tanta 21. La seconde caractéristique, le pluralisme juridique, est représentée par la coexistence du droit séculier, du droit canon $^{26}$ et du droit coutumier ${ }^{27}$.

${ }^{23}$ Le droit byzantin est divisé en trois périodes. La première s'étend du $\mathrm{VI}^{\mathrm{e}}$ au VIII $^{\mathrm{e}}$ siècles (codification du Corpus Iuris Civilis), la deuxième va du $\mathrm{IX}^{\mathrm{e}}$ siècle à la première moitié du XIII ${ }^{\mathrm{e}}$ siècle (codification des Basiliques) et la troisième de la seconde moitié du XIII ${ }^{\mathrm{e}}$ siècle à la Conquête de Constantinople (1453). La période qui nous intéresse plus particulièrement est naturellement la première. Voir I. Tsourka-Papastathi (1994), pp. 607-632.

${ }^{24}$ Au sujet du droit romain comme droit écrit, voir entre autres : Glenn (2000), pp.118-119 ; Watson (1995), pp. 469-476.

${ }^{25} \mathrm{Au}$ sujet du droit romain et de son évolution, voir, entre autres, Jolowicz (1972), Buckland (1950), et Kaser (1968).

${ }^{26}$ Le mode de production du canon diffère de celui du droit séculier. En effet, le canon est l'expression de la volonté d'une formation collégiale, c'est-à-dire un Concile ou un Synode. L'Édit de Milan (318), le Concile d'Ancyre (314), le Concile de Néocésarée (314-320) et le premier Concile œcuménique de Nicée (325) donneront à l'Église le pouvoir normatif en ce qui concerne son administration et ses fidèles. 
Dans les faits, le droit séculier et le droit canonique seront les principales sources du droit de l'empire. Ils auront une influence l'un sur l'autre, mais resteront distincts quant au fond et à la forme (I. Tsourka-Papastathi, 1994, p. 612).

Ainsi, l'influence principale qui a contribué à l'interdiction de rédiger des commentaires contenue dans Tanta 21 aurait son origine dans la tradition juridique romaine. Rappelons que Justinien a passé sous silence la traduction lors de la formulation du projet pour la nommer spécifiquement lors de la promulgation du Digeste. La question demeure donc, pourquoi interdire la traduction autre que littérale du latin vers le grec ? La réponse se trouve probablement dans le statut accordé au latin par Justinien ${ }^{28}$ et par Tribonien ainsi que par le désir de l'empereur de défendre et de préserver la culture latine et le droit romain classique (Honoré, 1978, p. 49; 1973-1974, p. 869).

Pour Justinien et Tribonien, le latin est la langue du droit par excellence; il doit donc être le réceptacle du droit de l'empire romain d'Orient et personnifier la culture juridique byzantine (Honoré, 19731974, p. 864). Dans cette optique, le droit se dit, se pense et s'énonce dans une langue et cette langue est le latin. Certes, l'enseignement du droit à Constantinople et à Beyrouth se donne en grec, car les étudiants connaissent mal le latin, mais ils sont tenus d'avoir une connaissance de base du latin, car il est la langue de l'administration et le droit menait à des postes élevés dans l'administration byzantine (p. 868). De plus, Tribonien, comme président de la commission chargée de compiler le Digeste, les Institutes et le second Codex, poursuivait quatre buts, c'est-à-dire : " to provide a handbook of practice for the bar of the higher courts and the provincial governors, to reform the law syllabus, to improve the quality of the recruits to government service, and to defend Latin culture » (p. 867). Ainsi, la défense de la culture passe par la langue qui est à son tour protégée par la règle de droit.

${ }^{27}$ On possède très peu d'information à propos du droit coutumier byzantin. Voir I. Tsourka-Papastathi (1994, pp. 611-612).

${ }^{28}$ La langue maternelle de Justinien aurait été, selon toute probabilité, le latin. De plus, il considère son empire comme la continuation de l'empire romain d'Occident et se voit comme un empereur romain dans le sens premier du terme. 
L'œuvre de compilation du Corpus Iuris Civilis a permis à Tribonien et à Justinien de préserver la culture juridique romaine, mais l'exercice aurait été futile si la traduction du Digeste n'avait pas fait l'objet de restrictions, car les praticiens du droit et les étudiants de langue grecque auraient eu tôt fait de traduire ou de faire traduire ce texte juridique et de se référer à la traduction et non pas à l'original ${ }^{29}$. On peut voir ici l'interaction entre droit, langue et idéologie. En effet, dans le cas de Tanta 21, la protection d'une langue et de sa culture juridique passe par l'interdiction de traduction autre que littérale du latin vers le grec.

\section{Conclusion}

Le droit, tout comme la traduction d'ailleurs, est porteur d'une idéologie qui s'exprime dans la formulation de la règle de droit. Il est le produit d'une société et d'une époque. En ce sens, la règle de droit énoncée dans Tanta 21 est particulièrement intéressante car elle associe une certaine forme de traduction, c'est-à-dire la traduction ultralittérale, à la protection d'une langue et d'une culture qui se trouvent être la culture et la langue du texte de départ. Ici, la traduction ultralittérale n'est pas porteuse d'assimilation de la langue ou de la culture d'arrivée. Bien au contraire, le but poursuivi est la protection du latin et de la culture " en voie d'extinction » qu'il représente. Ainsi, de la règle de droit qui impose une norme traductionnelle à l'idéologie sousjacente et aux conséquences de cette interdiction, le droit et la traduction ne sont jamais sans conséquence.

\section{Blake, Cassels \& Graydon s.r.l.}

${ }^{29}$ Les historiens du droit s'entendent pour dire que l'abandon par les universités de l'enseignement du droit dès la seconde moitié du $\mathrm{VI}^{\mathrm{e}}$ siècle est attribuable, entre autres, à l'impossibilité pour les étudiants de comprendre le latin. Voir entre autres: Scheltema (1970, p. 61) et Honoré (1973-1974, p. 868). De plus, Stein, sans donner de raison pour le choix de langue, affirme que la restriction de traduction imposée au CIC a été l'élément déterminant dans le peu d'intérêt dont la compilation de Justinien a bénéficié dans l'empire romain d'Orient (Stein, 1999, p. 35). Dans le même ordre d'idée, Honoré considère que le transfert de l'enseignement du droit de l'université d'Alexandrie au prétoire est la conséquence directe de l'interdiction de rédaction de commentaires et de la restriction de traduction (Honoré, 19731974, p. 868). Michel Morin, de son côté, considère que la raison pour laquelle l'œuvre de Justinien sera peu utilisée dans l'empire romain d'Orient est que cette compilation est anachronique (Morin, 2004, p. 79-80). 


\section{Références}

BARTHÉLEMY, Dominique (1963). Les devanciers d'Aquila : première publication intégrale du texte des fragments du Dodécaprophéton trouvés dans le désert de Juda, précédée d'une étude sur les traductions et recensions grecques de la Bible réalisées au premier siècle de notre ère sous l'influence du rabbinat palestinien. Supplements to Vetus Testamentum. v. 10, Leiden, E.J. Brill.

BERGER, Adolf (1945). "The Emperor Justinian's Ban upon Commentaries of the Digest ». Quarterly Bulletin of the Polish Institute of Art and Sciences in America III, April-June, pp. 656-696, également publié avec correction dans : BIRD Suppl. Post-Bellum, 1948, pp. 124169.

BROCK, Sebastian (1979). "Aspects of Translation Technique in Antiquity ». Greek, Roman, and Byzantine Studies, 20 (Spring), pp. 6987.

BUCKLAND, W. (1950). A Text-Book of Roman Law from Augustus to Justinian. $3^{\mathrm{e}}$ édition révisée par Peter Stein. Cambridge, Cambridge University Press.

CHORUS, Jeroen M.J. (1993). «L'enseignement du droit romain en Occident de 250 à 500 : essai de tableau ». Revue d'histoire du droit, fascicule 3, pp. 195-204.

CICERO (1949). De inventione, De optimo genere oratorum, Topica. Édité et traduit par H. M. Hubbell. Loeb Classical Library, Cambridge, Harvard University Press.

- (1989). « The Fortunes of Non Verbum pro Verbo: or, why Jerome is not a Ciceronian ». The Medieval Translator, Cambridge, Brewer, pp. 15-35.

COPELAND, Rita (1991). Rhetoric, Hermeneutics, and Translation in the Middle Ages: Academic Traditions and Vernacular Texts. Cambridge, Cambridge University Press.

DE ROBERTIS, Antonio (1984). La interpretazione del Corpus iuris in Oriente $e$ in Occidente: approcio comparativo alle posizioni 
ermeneutiche degli scoliasti bizantini e della glossa di Accursio. Naples, Jovene.

Digesta Iustiniani Augusti (1963). (Theodor Mommsen, éditeur), Berolini, Weidmann.

EDEN, Kathy (1997). Hermeneutics and the Rhetorical Tradition. Chapters in the Ancient Legacy \& the Humanist Reception. New Haven and London, Yale University Press.

GAUDEMET, Jean (2000). Les Institutions de l'Antiquité. Collection Domat droit public, Paris, Éditions Montchrestien.

GAUDEMET, Jean (2000a). Droit privé romain. $2^{\mathrm{e}}$ édition. Paris, Montchrestien.

GLENN, Patrick H. (2000). Legal Traditions of the World. Oxford, Oxford University Press.

HARL, Marguerite, Gilles DORIVAL et Olivier MUNNICH (1988). La Bible grecque des Septante. Du judaïsme héllénistique au christianisme ancien. Paris, Éditions du Cerf.

HONORÉ, A.M. (1973-1974). « The Background to Justinian’s Codification ». Tulane Law Review, vol. 48, pp. 859-893.

HONORÉ, A.M. et Tony HONORÉ (1978). Tribonian. Ithaca, Cornell University Press.

IRVINE, Martin (1994). The Making of Textual Culture. "Grammatica » and Literary Theory, 350-1100. Cambridge, Cambridge University Press.

I. TSOURKA-PAPASTATHI, Despina (1994). « La réception du droit musulman par les systèmes de l'Europe byzantine ». La réception des systèmes juridiques: Implantation et destin, (sous la direction de Michel Doucet et Jacques Vanderlinden), CICLEF, Bruxelles, Bruylant, pp. 607-632.

JOLOWICZ, H. F. (1972). Historical Introduction to the Study of Roman Law. Cambridge, Cambridge University Press. 
KASER, M. (1968). Roman Private Law. $2^{\mathrm{e}}$ édition, R. Dannenbring, traducteur. London, Butterworths.

KELLY, Louis G. (1977). "Linguistics and Translation in Saint Augustine ». The Bible Translator, 24, pp. 134-139.

MACLEAN, Ian (1992). Interpretation and Meaning in the Renaissance. The Case of Law. Cambridge, Cambridge University Press.

MORIN, Michel (2004). Introduction historique au droit romain, au droit français et au droit anglais. Montréal, Éditions Thémis.

O'CONNELL, Kevin G. (1972). The Theodotionic Revision of the Book of Exodus: A Contribution to the Study of the Early History of the Transmission of the Old Testament in Greek. Cambridge, Harvard University Press.

ORIGEN (1875). Hexaplorum quae supersunt; sive, Veterum interpretum Graecorum in totum Vetus Testamentum fragmenta. Post Flaminium Nogilium, Drusium, et Montefalconium, adhibita etiam versione syro-hexaplari, concinnavit, emendavit, et multis partibus auxit Fridericus Field. Oxonii E typographeo Clarendoniano.

PRINGSHEIM, F. (1950). « Justinian's Prohibition of Commentaries to the Digest». Revue internationale des droits de l'Antiquité, IV, pp. 383-415.

SARCEVIC, Susan (1997). New Approach to Legal Translation. The Hague, Kluwer Law International.

SCHELTEMA, H. (1970). L'enseignement du droit des antécesseurs. Leiden, Brill.

SCHRADER, H.E.S (1832). Justinien $1^{\text {er }}$, empereur. Corpus Iuris Civilis. Recensuit Eduardus Schrader. Tomus 1. Institutiones libri IV, Berlin.

STEIN, Peter (1999). Roman Law in European History. Cambridge, Cambridge University Press. 
The Digest of Justinian. Translation edited by Alan Watson (1985). Philadelphia, University of Pennsylvania Press.

The Visigothic Code (Forum judicum) (1982). Traduit et édité par S.P. Scott. Littleton, F.B. Rothman.

TROJE, H.-E. (1971). Graeca laguntur. Frankfurt/Main.

VAN DEN BERGH, G.C.J.J. et B.H. Stolte Jr (1977). «The Unfinished Digest Edition of Henrik Brenkman (1681-1736)». Revue d'histoire du droit, v. 45, pp. 227-306.

VAN DER WAL, Nicolaas (1953). Les commentaires grecs du Code de Justinien. 's-Gravenhage, Vitgeverij, Excelsior.

VERMER, Hans J. (1988). « From Cicero to Modern Times : Rhetorics and Translation ». Translation Theories - Grundlagen und Standorte, Justa Holz-Mänttäri (éditeur), Tampere, Studia Translatologica A.1, pp. 93-128.

VISMARA, G. (1992). " Il diritto nel regno dei Goti ». Teodorico II Grande ei Goti d'Italia, Atti del XIII Congresso internationale di studi sull'alto Medioevo, Milan, pp. 275-315.

WARD, John O. (1995). Ciceronian Rhetoric in Treatise, Scholion and Commentary. Fascicule 58, Typologie des sources du Moyen Âge occidental, Institut d'études médiévales. Turnhout, Belgique, Université Catholique de Louvain.

WATSON, Alan (1988). Failure of the Legal Imagination. Philadelphia, University of Pennsylvania Press.

WATSON, Alan (1995). «From Legal Transplants to Legal Formants ». American Journal of Comparative Law, vol. 43, no 3, pp. 469-476.

WENGER, Leopold (1953). Die Quellen des römischen Rechts. Wien, A. Holzhausen.

WHITMAN, James Q. (1991). "A Note on the Medieval Division of the Digest ». Revue d'histoire du droit, v. 59, pp. 269-284. 
WINKEL, Laurens (1997). « Le droit romain et la philosophie grecque, quelques problèmes de méthode ». Revue d'histoire du droit, fascicule 3, pp. 373-384.

YUHONG, Zhao (2000). "Legal Translation in the Legislative Genre ». Fan yi xue bao / Journal of Translation Studies, vol. 4, pp. 1944.

RÉSUMÉ : Droit, traduction, langue et idéologie : Kata poda ou la traduction pas à pas selon Justinien $\mathbf{1}^{\text {er }}$ - Cet article porte sur la Constitutio Tanta 21 qui interdit la rédaction de commentaires si ce n'est la traduction ultra-littérale du latin vers le grec qui frappe le Digeste de Justinien. Il débute par une analyse de la teneur et de la portée de Tanta 21, puis il s'intéresse aux divers facteurs qui ont pu participer à la formulation de cette interdiction, c'est-à-dire les principes appliqués à la traduction biblique, l'influence de la tradition juridique romaine et finalement le statut du latin comme langue juridique par excellence.

ABSTRACT: Law, translation, language, and ideology: Kata poda or translation step by step according to Justinian the $\mathbf{1}^{\text {st }}-$ This article examines Constitutio Tanta 21 which prohibits all commentary on Justinian's Digest, except for ultra-literal translation from Latin to Greek, or what the Greeks call kata poda. It begins by analyzing the terms and significance of Tanta 21, and it then tries to circumscribe the various factors that might have contributed to the formulation of the prohibition, namely the principles applied to Bible Translation, the influence of the Roman legal tradition, and finally, the importance of Latin as the language of the law.

Mots-clés : Digeste de Justinien, Tanta 21, traduction juridique, traduction ultra-littérale, idéologie et langue.

Keywords: Justinian’s Digest, Tanta 21, legal translation, ultra-literal translation, ideology and language.

Claire-Hélène Lavigne : Blake, Cassels \& Graydon s.r.l. 600, boulevard de Maisonneuve Ouest, bureau 2200

Montréal (Québec) H3A 3J2

Courriel : Claire-Helene.Lavigne@blakes.com 